\title{
Norois
}

Environnement, aménagement, société

\section{Rétablir la libre circulation piscicole dans les vallées fluviales : mise en perspective des enjeux et des aménagements à partir du cas de l'Aulne (XIXe- XXIe siècles)}

Restoring free fish migration in the river valleys. An historical point of view

(19th-21th centuries) of dam management on the Aulne river (Brittany, France)

\section{Caroline Le Calvez}

\section{(2) OpenEdition}

Journals

Édition électronique

URL : https://journals.openedition.org/norois/5779

DOI : 10.4000/norois. 5779

ISBN : 978-2-7535-5086-5

ISSN : 1760-8546

Éditeur

Presses universitaires de Rennes

Édition imprimée

Date de publication : 31 décembre 2015

Pagination : 33-50

ISBN : 978-2-7535-5084-1

ISSN : 0029-182X

Référence électronique

Caroline Le Calvez, «Rétablir la libre circulation piscicole dans les vallées fluviales : mise en perspective des enjeux et des aménagements à partir du cas de l'Aulne (XIXe-XXIe siècles) », Norois [En ligne], 237 | 2015, mis en ligne le 31 décembre 2017, consulté le 28 janvier 2022. URL : http:// journals.openedition.org/norois/5779; DOI : https://doi.org/10.4000/norois.5779 


Presses
Universitaires
de Rennes
www.pur-editions.fr

\title{
Rétablir la libre circulation piscicole dans les vallées fluviales : mise en perspective des enjeux et des aménagements à partir du cas de l'Aulne ( $\mathrm{XIX}^{\mathrm{e}}-\mathrm{XXI}^{\mathrm{e}}$ siècle)
}

\author{
Restoring Free Fish Migration in the River Valleys \\ An Historical Point of View (19 $9^{\text {th }}-21^{\text {th }}$ Centuries) of Dam Management on the Aulne River \\ (Brittany, France)
}

\author{
Caroline Le CALvez
}

ESO - UMR 6590 CNRS, Université Rennes 2, Place du Recteur-Henri-Le-Moal, CS 24307 - 35043 RenNes
cedex. (caroline.lecalvez@uhb.fr)

Résumé : La continuité écologique est emblématique de la gestion écologique des cours d'eau. En Bretagne, l'effacement des ouvrages transversaux répond aux objectifs de restauration des populations de poissons migrateurs et de rétablissement de la continuité écologique pour atteindre le bon état écologique exigé par la Directive Cadre sur l'Eau. Le propos vise à mettre en perspective l'articulation entre l'effacement des ouvrages et la remontée des poissons migrateurs à partir de l'exemple de l'Aulne, rivière reconnue historiquement pour sa fréquentation par le saumon atlantique. La mobilisation d'archives et d'un corpus documentaire apporte un éclairage sur la trajectoire de gestion et d'aménagement des ouvrages hydrauliques. L'article illustre l'évolution de la conception de la rivière depuis le $\mathrm{XIX}^{\mathrm{e}}$ siècle et les difficultés d'ancrage des nouvelles normes de qualité environnementale, mises en évidence à travers l'étude d'une expérimentation controversée du rétablissement temporaire de la continuité écologique.

\begin{abstract}
Ecological continuity represents a specific priority for rivers management. In Brittany (France), dam removal is intended to restore free movement of migratory fish and to provide ecological continuity. This objective of ecological continuity is itself intended to recover a favorable ecological status, ensuring the quality of water required by the EU Water Framework Directive. Based on the case of Aulne River, which is historically known to be highly frequented by Atlantic salmon, this article put the link between dam removal and free movement of migratory fish into historical perspective. A thorough analysis of archives and documentary evidences allows us to shed light on the evolution of management and techniques for the restoration of salmon runs. We illustrate the evolution of river's concept since the mid-nineteenth century and discuss various challenges associated with experimental policy of temporary restoration of ecological continuity.
\end{abstract}

Mots clés : continuité écologique - pêche - DCE - aménagement fluvial - controverse - approche diachronique

Keywords : ecological continuity - fishery - Water Framework Directive - hydraulic engineering - controversy - diachronic approach 


\section{INTRODUCTION}

Les espaces fluviaux de l'ouest français sont depuis une vingtaine d'années au cœur de logiques de gestion patrimoniale parfois antagonistes (Germaine et Barraud, 2013; Barraud et Portal, 2013). Dans les années 1990, les actions de mise en valeur des nombreux aménagements, hérités de la vocation productive des vallées fluviales, entrent en concurrence avec la demande sociale de valorisation du patrimoine naturel. La gestion écologique des cours d'eau se développe et s'appuie sur l'application du concept de la restauration écologique (Clewell et Aronson, 2010; Morandi, 2014). Le paysage de la rivière sauvage, qui divague, libérée des aménagements anthropiques, est une référence vers laquelle tend le champ de la gestion de l'eau et des milieux aquatiques; et que la continuité écologique des cours d'eau, c'est-à-dire la libre circulation des organismes vivants, le bon transport des sédiments et l'assurance du bon fonctionnement des réservoirs biologiques (ONEMA, 2010) est censée garantir. Les premières expériences d'effacement d'ouvrages hydrauliques (seuils, barrages) sont mises en place dans l'objectif de retrouver une rivière naturelle (Barraud, 2011). Inscrit à l'annexe V de la Directive Cadre sur l'Eau (2000/60/CE), le concept de continuité s'impose progressivement en France pour atteindre la qualité écologique exigée par le texte européen. Face aux attentes d'améliorations hydromorphologiques et biologiques, les ouvrages implantés parfois très anciennement deviennent de potentiels obstacles à la « libération » longitudinale et latérale des cours d'eau. Leur mise en conformité par des opérations d'effacement de l'effet seuil est appuyée par un cadre législatif et réglementaire renforcé depuis 2006 (Germaine et Barraud, 2013). Les prescripteurs de la gestion des poissons migrateurs s'emparent de la mesure pour défendre le repeuplement de populations autonomes de poissons migrateurs dans les cours d'eau et notamment des stocks de saumon atlantique (Salmo salar L.), espèce symbole de la qualité de l'eau (Laurent, 1995). Pour enrayer la disparition des saumons de la plupart des grands réseaux hydrographiques français, l'effacement d'ouvrages transversaux est préconisé. En effet, leur implantation entrave la remontée de ces poissons migrateurs vers les zones de reproduction situées dans les parties amont des bassins. Elle est considérée comme la cause principale de la raréfaction de l'espèce dans les cours d'eau (Chaney, 1995 ; Croze, 2008). Les modifications opérées sur les ouvrages transversaux relèvent donc d'un croisement des enjeux et des justifications et sont citées aussi bien parmi les outils de planification et de programmation de la gestion de l'eau et des milieux aquatiques, que parmi ceux relatifs à la gestion des poissons migrateurs, ou à la préservation de la biodiversité et des paysages. L'ambition des exigences en matière d'amélioration des milieux et de limitation des impacts humains sur l'environnement font des aménagements d'ouvrages un outil privilégié de l'action publique française pour la préservation et la restauration de la qualité environnementale dans les espaces fluviaux.

Devenu une action incontournable, l'effacement des ouvrages transversaux n'en est pas moins un outil récent. Notre propos vise ainsi à replacer cette mesure au sein de la dynamique de gestion des espaces fluviaux par une mise en perspective historique de la gestion de ces aménagements anthropiques. La problématique de la libre circulation des poissons migrateurs, articulée aux enjeux de la restauration des populations de poissons migrateurs et de la restauration de la continuité écologique pour l'atteinte des objectifs européens de qualité de l'eau, a contribué à construire l'action d'effacement comme une solution privilégiée, qui occulte des mesures d'équipement des ouvrages, anciennement utilisées pour favoriser la remontée des migrateurs. La perception nouvelle des ouvrages vus comme des contraintes au bon fonctionnement des milieux, illustre la suprématie d'un nouveau principe d'aménagement construit sur la logique écologique au détriment de la prise en compte des liens entre la société locale et le cours d'eau.

Avec 25 cours d'eau fréquentés par le saumon atlantique, la Bretagne est la région française où les populations de saumon atlantique sont les mieux préservées (Arago et Vauclin, 2000). Choisi pour notre démonstration, le fleuve côtier Aulne est historiquement connu comme fleuve à saumons (Rainelli et Thibault, 1980). La partie aval du cours d'eau a été canalisée au XIX ${ }^{\mathrm{e}}$ siècle lors de la construction du Canal de Nantes à Brest. Cet objet géographique illustre particulièrement bien les phases et les inflexions qui mènent à une considération contemporaine inédite pour l'effacement des ouvrages et la 
ressource piscicole salmonidée, entendue comme garante de la qualité de l'eau, et tendant à occulter les autres dimensions de la continuité écologique comme la problématique de la circulation sédimentaire. Une analyse qualitative de documents d'archives couvrant la période entre le XIX ${ }^{\mathrm{e}}$ siècle et les années 1950, ainsi que l'étude de documents institutionnels (stratégiques, programmatiques et opérationnels) pour la période post-1960 ont été menées. Elles ont permis de retracer l'évolution des modalités d'intervention sur les aménagements fluviaux pour la restauration des populations de saumon atlantique, et apportent un éclairage sur les impacts socio-spatiaux de l'application du concept de continuité écologique des cours d'eau.

La première partie de l'article opère un retour historique sur les premières opérations de restauration de la libre circulation du saumon atlantique sur l'Aulne (milieu du XIX e siècle-années 1930). Ces actions destinées à favoriser la remontée de l'espèce reposent fortement sur des mesures d'adaptation et d'équipement des barrages et ont pour vocation de développer la ressource saumon dans un objectif économique, dans un jeu entre fermiers locaux et services de l'État. La deuxième partie aborde la période postérieure à la Seconde Guerre mondiale et inaugurée par la loi sur l'eau de 1964. L'émergence, puis la domination des critères écologiques de bonne qualité de l'eau dans l'encadrement juridique et dans les programmes stratégiques, engendre une évolution notable de l'action publique. Sur plusieurs cours d'eau bretons, dont l'Aulne, l'enjeu de la remontée du saumon retrouve une nouvelle vigueur dans des politiques publiques qui s'appuient sur des effacements d'ouvrages, les dispositifs de franchissements de la période précédente n'ayant pas donné les résultats escomptés. Si l'aménagement des ouvrages pour la remontée des migrateurs est une constante sur le territoire étudié, sa justification et ses modalités évoluent en fonction du sens donné à la présence des salmonidés et de la domination progressive des enjeux écologiques. Aujourd'hui, dans le cadre de la mise en œuvre d'une gestion écologique des cours d'eau, l'effacement des ouvrages pose la question de l'existence d'un projet de territoire, discussion que nous ouvrirons en troisième partie à partir d'une expérimentation controversée réalisée sur l'Aulne. En effet, un effacement temporaire et expérimental des ouvrages, que cer- tains acteurs considèrent comme l'aboutissement logique de plus d'un siècle d'actions en faveur de la remontée du saumon, suscite de vives oppositions d'usagers locaux.

\section{FAIRE REMONTER LE SAUMON SUR L'AULNE : UN ENJEU DE DÉVELOPPEMENT LOCAL

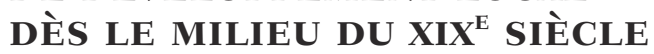

Le dépeuplement des cours d'eau en saumons est une problématique à laquelle les acteurs locaux et administratifs tentent de faire face dès le $\mathrm{XIX}^{\mathrm{e}}$ siècle. Si le repeuplement artificiel est la réponse la plus ancienne mise en œuvre pour aider à la protection de l'espèce (Breton, 1993), elle est progressivement couplée à des mesures en faveur de la remontée des saumons vers les zones de frayères afin de favoriser la reproduction naturelle (Thomas, 2009; Thibault et Vinot, 1989). En effet, la particularité des rivières à saumons est l'ancienneté des actions en faveur de la libre circulation de l'espèce (Barraud, 2011). La loi du 31 mai 1865 introduit l'obligation d'équiper les barrages nouveaux en échelles à poissons (ONEMA, 2011). Des campagnes d'équipement des ouvrages en dispositifs de franchissement voient le jour et contribuent à faire de la libre circulation une action privilégiée sur l'Aulne. Ces actions, certes prémices du principe de la continuité écologique, sont avant tout menées au nom du développement local de la pêche et du tourisme. L'analyse de documents d'archives de l'Administration d'État apporte un éclairage sur les temporalités de l'action et les choix locaux relevant d'une conception économique et touristique du territoire et de l'espèce.

\section{Une articulation progressive entre repeuplement et échelles à poissons}

Le corpus analysé est constitué d'une centaine de documents (rapports, courriers, avis, arrêtés et règlements) conservés aux Archives départementales du Finistère. Ils y ont été versés par la Préfecture, le Service des voies navigables relevant de l'Administration des Ponts et Chaussées et la Direction départementale de l'Équipement du Finistère. Les documents appartiennent à la sous-série «Navigation intérieure : fleuves et canaux » (3S) issue de la série $\mathrm{S}$ « Travaux publics et transports » 
et aux suppléments (29S). La période couverte par l'analyse commence à la mise en service de la partie finistérienne du canal de Nantes à Brest en 1834. Si les données restent ponctuelles jusque dans les années 1840, on assiste dans la deuxième moitié du siècle à un foisonnement des données produites par l'Administration dans le cadre de la gestion finistérienne du canal. Un travail de dépouillement systématique des dossiers a été mené pour sélectionner les documents. Les données recueillies ont permis de relever les caractéristiques principales de la mise en œuvre d'une gestion de la ressource « poissons migrateurs » et ses justifications.

L'Aulne est un fleuve côtier anciennement aménagé par les populations locales pour la pêche au saumon (pêcheries) et l'utilisation de la force hydraulique (moulins). À partir des années 1810, la canalisation de l'aval de l'Aulne sur près de $70 \mathrm{~km}$ fait disparaître les habitats favorables à la reproduction du saumon et transforme les conditions d'accès aux sites de frai situés à l'amont sur l'Aulne rivière (figure 1) (Croze et al, 2001).

Le rehaussement du niveau d'eau pour la navigation est réalisé par l'implantation de 28 barrages, chacun équipé d'une écluse destinée au passage des bateaux, d'un déversoir droit ou en « $V$ » et d'un pertuis pour l'évacuation de l'eau notamment lors des vidanges d'entretien (figure 2).

Un premier projet local de construction d'échelles à poissons ${ }^{1}$ émerge au début des années 1860, donnant lieu à une première controverse avec les services de l'État ${ }^{2}$. Il naît de la volonté d'un acteur local amodiataire $^{3}$ de la pêche sur l'Aulne canalisée qui explique vouloir aider à la remontée des saumons en construisant des dispositifs sur tous les barrages du lot dont il est fermier pour développer la pêche et réaliser des bénéfices sur les permissions de pêche. Ce premier projet se heurte à l'Administration qui reconnaît l'intérêt de la remontée des saumons mais remet en cause les échelles à poissons,

1. Dans cette première partie, le choix a été fait de reprendre le terme d'échelle à poissons qui est dominant dans les documents d'archives analysés. Pour les périodes postérieures aux années 1950 la dénomination évolue en même temps que le degré de technicité de ce système de franchissement, le terme de passe à poissons s'impose et sera utilisé pour la suite du propos.

2. Le corps des Ponts et Chaussées est en charge du Service du canal qui relève du ministère des Travaux Publics.

3. L'État est propriétaire du canal de Nantes à Brest, il afferme les droits de pêche et de chasse. Le droit de pêche est fait par amodiation de lots déterminés par le Service du canal. L'amodiataire accorde ensuite des permissions de pêche sur le linéaire qu'il a en fermage. considérées comme des dispositifs très onéreux et à l'efficacité incertaine. De plus, pour les ingénieurs des Ponts et Chaussées ce ne sont pas les ouvrages du canal qui sont responsables du déclin des saumons mais la pêche en estuaire et le braconnage ${ }^{4}$ qui détruisent la ressource. Par la suite, le Conseil général du Finistère émet le vœu en 1889 et en 1904 d'aider à la reproduction naturelle de l'espèce en finançant pour partie des échelles à poissons. La dynamique de généralisation des équipements sur les ouvrages de l'Aulne (Thomas, 2009) est lancée en 1903 à l'initiative d'un notable, M. De Kerjégu. Cumulant les fonctions de député, de président du Conseil général et d'amodiataire de lots de pêche sur l'Aulne, il est doublement intéressé par l'équipement des ouvrages et la valorisation économique de la pêche. Il débloque la situation en lançant des projets sur 5 barrages $^{5}$, l'État s'engageant financièrement sur la moitié de l'investissement, et l'Administration soutenant la campagne d'équipement.

\section{L'activité de pêche au saumon au cœur d'un projet de développement local}

L'activité de pêche a pâti de la construction du canal et des pollutions engendrées par l'extraction minière et les productions qui utilisaient l'eau (Cambry, 1993; Thibault, 1995; Thomas, 2009). À la fin du XIX ${ }^{\text {e }}$ siècle, trois types de pêches se côtoient sur l'Aulne : la pêche de subsistance, la pêche commerciale et la pêche sportive. Les premiers projets de construction d'échelles à poissons relèvent d'une volonté de développer l'activité de pêche commerciale. La valorisation économique de la ressource en salmonidés pour le développement local et l'amélioration de la vie des populations est l'objectif principal des politiques de repeuplement. Pour l'ensemble des acteurs, l'image de marque de l'Aulne est associée à sa fréquentation par le saumon. Le canal doit retrouver « son ancienne réputation au point de vue de la pêche des salmonidés ${ }^{6}$ ». Rainelli et Thibault (1980) postulent que l'enjeu du repeuplement s'est construit en partie sur le mythe de l'abondance ancienne en saumons de l'Aulne. La crainte de la disparition de l'espèce repose en

\footnotetext{
4. AD29, 3S62, rapport de l'ingénieur ordinaire daté du 8 janvier 1900 et procès-verbal des délibérations du Conseil général du Finistère daté du 4 mai 1905.

5. AD29, 29S40.

6. AD29, 3S62, rapport de l'ingénieur ordinaire, 28 janvier 1905.
} 


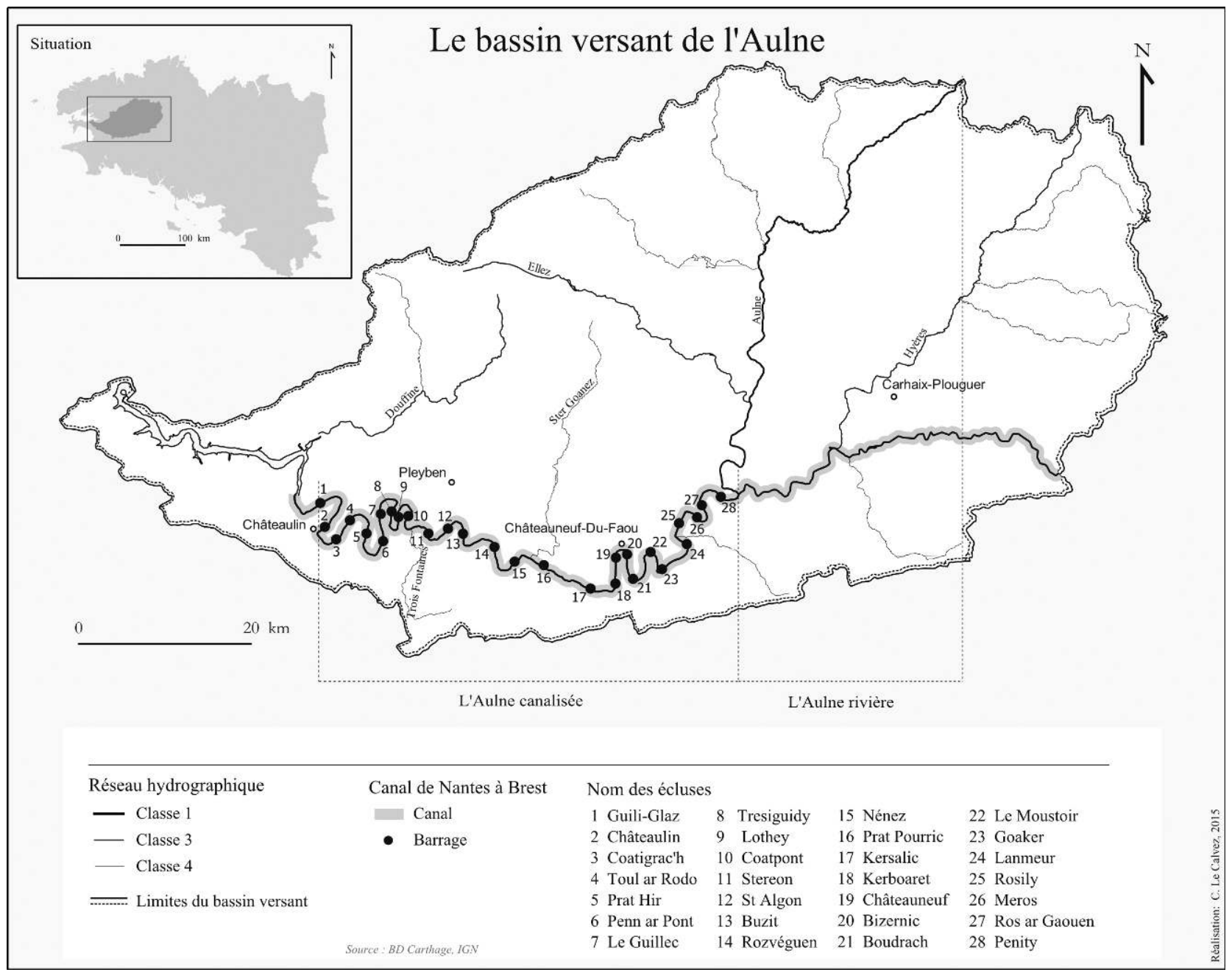

Figure 1: Carte du bassin versant de l'Aulne : localisation du tronçon canalisé et des barrages Map of the Aulne watershed: location of channeled section and of dams

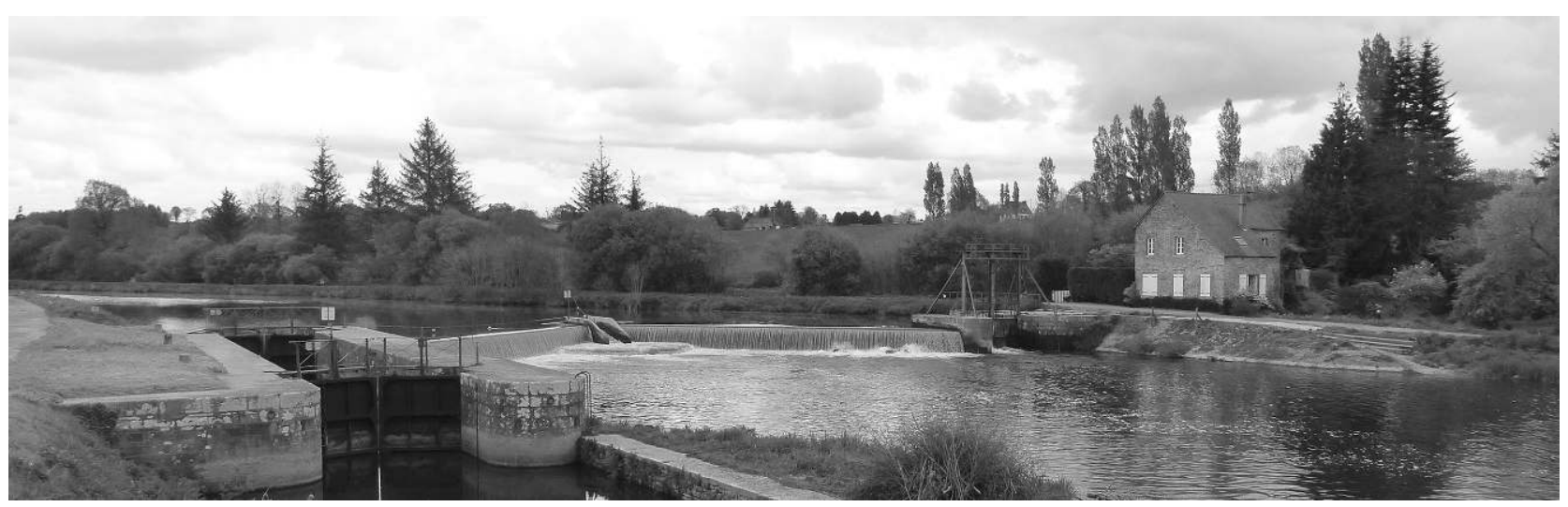

Figure 2 : Le barrage de Goaker. De gauche à droite : écluse, déversoir équipé d’une passe à poisson et pertuis (Crédits : C. Le Calvez, 2014) Goaker dam. From left to right : sluice, dam with a fishway and opening (credits : C. Le Calvez, 2014) 
particulier sur un discours idéologique accusant la proclamation de la liberté de pêche "pour tous et sur tous les cours d'eau » lors de la Révolution française d'engendrer des prélèvements abusifs sur la ressource (Breton, 1993). Initialement tourné vers le passé à travers une reconstruction mythifiée d'un territoire riche et prospère grâce au saumon, l'argumentaire glisse peu à peu vers le développement du territoire. Cette visée développementaliste est d'abord portée par le Conseil général puis par des acteurs spécialisés comme la Fédération finistérienne des sociétés de pêche à la ligne. Avec la saisie de l'enjeu par le Conseil général, c'est l'ensemble de l'activité pêche qui est directement concernée. Le retour des saumons permettrait en effet d'améliorer l'alimentation publique en apportant aux populations locales des compléments de nourriture, de développer la pêche commerciale du saumon, de rendre l'activité de pêche sportive plus attractive et d'augmenter le prix de l'amodiation c'est-à-dire assurer un revenu supplémentaire à l'État. Progressivement, l'enjeu se déplace d'un type de pêche à un autre pour se fixer sur la pêche sportive. Le tourisme de la pêche devient alors la justification majeure dans l'Entre-deux-guerres. Localement, les acteurs voient se développer « un certain mouvement touristique que les chemins de fer vont chercher à développer par une propagande en France et à l'étranger ${ }^{7}$ » dans un contexte de prise en main de la gestion piscicole par les Sociétés de Pêche et leur représentation départementale. La pêche à la ligne s'impose au détriment des pêches alimentaire et économique. La domination de cette pratique renouvelle l'approche de la remontée des saumons; des échancrures, nouveaux types de dispositifs de franchissement, sont testées. En parallèle, les actions mises en œuvre pour restaurer le stock sont de plus en plus orientées vers un durcissement des conditions de pêche dans le cadre de la réglementation locale. L'utilisation des filets de pêche dans l'Aulne est interdite. En 1922, le cours d'eau est classé en " cours d'eau à migrateurs » au titre du décret du 31 janvier 1922 qui dresse la liste des cours d'eau bretons sur lesquels la libre circulation des espèces migratrices doit être garantie. La possibilité de faire de l'Aulne

7. AD29, 29S44, courrier du Président de la Fédération finistérienne des sociétés de pêcheurs à la ligne, années 1920-1930. canalisée une réserve pour le saumon est évoquée ${ }^{8}$ pour accélérer la modification des pratiques de pêche et empêcher le braconnage.

\section{La politique d'équipement en dispositifs de franchissement}

Les premiers initiateurs privés, qui voyaient dans l'équipement un intérêt pour le développement de l'amodiation des lots de pêche, laissent progressivement la place à des projets d'ampleur initiés par l'assemblée départementale (conseil général) et l'État, puis par la Fédération des Sociétés de pêcheurs à la ligne. Le processus de décision est complexe et administrativement lourd (Brunot et Cocquand, 1982). Chaque projet entraîne un aller-retour constant entre l'échelon local où interviennent les ingénieurs du canal, le niveau départemental incarné par le préfet, et le pouvoir central où la décision finale est prise par le ministère des Travaux Publics. Chaque étape du projet d'équipement fait l'objet de rapports, d'examens, de modifications et de validations. Les ingénieurs et conducteurs des Ponts et Chaussées ont un rôle central. Ils formulent des avis sur les demandes des initiateurs, conçoivent les projets et déterminent les conditions techniques et financières de leur réalisation. En tant que représentant exécutif de l'assemblée départementale et du pouvoir central à partir de 1871 le Préfet est un relais incontournable entre les acteurs (Minot, 2000).

L'appréhension de l'enjeu de la remontée est d'abord ponctuelle. Les premiers projets visent l'amélioration des conditions de franchissement aux barrages les plus en aval comme ceux de Châteaulin et Coatigrac'h dans les années 1860, Guili Glaz autour de 1888, et les plus problématiques notamment à cause de la hauteur de saut comme Prat Hir (1904) et Prat Pourric (1904). La politique d'équipement s'étend à l'ensemble du linéaire du cours d'eau canalisé lorsque les acteurs publics s'emparent du problème. Elle succède à une problématisation au cas par cas, à l'échelle du barrage. La systématisation de l'aménagement des barrages qui caractérise l'intervention au début du $\mathrm{Xx}^{\mathrm{e}}$ siècle témoigne d'une perception nouvelle de l'enjeu. En 1905, le projet d'équipement qui voit le jour sur toute la partie

8. AD29, 29S44, rapport de l'ingénieur des TPE subdivisionnaire, 29 décembre 1939. 
finistérienne du canal de Nantes à Brest (Aulne et Hyères) prévoit chaque année l'équipement de deux barrages, en priorisant les barrages dont les hauteurs de chute sont les plus pénalisantes pour les saumons. Entre 1906 et 1919, ce sont 14 échelles à poissons qui sont mises en place sur l'Aulne. Dans les années 1930, une nouvelle méthode de remontée des migrateurs est expérimentée sur l'Aulne. La Fédération de Pêche fait installer des échancrures dans les déversoirs de certains barrages. Ces dispositifs créent un appel pour les saumons et facilitent le passage de l'obstacle. Ils remplacent ou complètent l'équipement en échelles à poissons construites avant 1920, jugées vétustes et peu efficaces. Cette évolution est le marqueur d'une adaptation du mode d'intervention publique à la problématique de la remontée de l'espèce. L'articulation entre le repeuplement en saumons et le rétablissement de la libre circulation relève bien de choix politiques et techniques territorialisés liés à une évolution des porteurs de projets et de la perception de l'enjeu.

De manière synthétique, trois périodes se succèdent sur l'Aulne. La première période (1850-fin $\mathrm{XIX}^{\mathrm{e}}$ siècle) est celle des initiatives individuelles et ponctuelles qui contribuent à faire de l'équipement des barrages une solution privilégiée à la remontée des saumons. Les essais, les aménagements ponctuels, les témoignages de leur efficacité dans les rapports sont autant de faits qui font évoluer la perception qu'ont les acteurs de ces dispositifs. La deuxième période (1906-1920) est marquée par la reconnaissance par l'ensemble des acteurs de l'enjeu et la mise en œuvre d'une politique locale tournée vers le développement du territoire. L'extension spatiale des campagnes d'équipement ainsi que l'accompagnement financier des projets par l'administration du canal sont les révélateurs d'une véritable action publique en faveur des saumons. De 1921 à la fin des années 1940, les modalités évoluent vers un équipement ponctuel en dispositifs de types échancrures. À cette même période, la raréfaction du transport fluvial sur la partie finistérienne du canal est une opportunité pour le développement du tourisme pêche. Cependant, malgré la politique d'équipement en échelles à poissons et échancrures, de repeuplement artificiel et la réglementation accrue des pratiques de pêche, la problématique du peuplement en saumon n'est pas résolue. Pour pallier cet échec et garantir le développement de la pêche, une mesure radicale de «suppression de toutes les installations du canal [pour favoriser] le rétablissement de l'Aulne dans son état naturel ${ }^{9}$ " est évoquée dans la période d'après-guerre par les ingénieurs du canal. Cette première proposition d'un retour à une rivière naturelle n'est cependant pas réalisée.

La politique d'intervention en faveur de la remontée des saumons est dépendante de la vocation économique des ouvrages. L'Aulne canalisée est un axe réglementé et géré par l'Administration qui se distingue de la vallée de l'Aulne par ses activités, ses codes et la politique qui s'y applique. En se proposant d'aider à la remontée des migrateurs, les acteurs participent au rattachement de l'infrastructure fluviale économique à la vallée. Alors que le déclin du canal dû à la concurrence avec les autres voies de transports (réseaux ferrés et routiers) est entamé, le projet de développement touristique de la pêche appuyé sur le renouveau de la ressource en saumons contribue à la formulation d'un nouveau projet de développement local.

\section{L'ARTICULATION ENTRE REPEUPLEMENT BIOLOGIQUE ET QUALITÉ ÉCOLOGIQUE : DE NOUVELLES MODALITÉS D'AMÉNAGEMENT DES OUVRAGES}

Radié des voies navigables en 1957, le tronçon finistérien du canal de Nantes à Brest perd sa vocation économique première. À cette rupture dans la gestion du cours d'eau coïncide la reconnaissance de l'activité de la pêche au saumon comme une activité touristique locale majeure. L'évolution du stock de saumons est quantifiée annuellement à partir des relevés de captures de saumons à la ligne. Le constat d'un effondrement des stocks de saumons à partir des années 1960-1970 est dressé sur les différents réseaux hydrographiques français dont celui de l'Aulne. Une nouvelle forme d'action publique émerge pour protéger et restaurer la ressource, caractérisée par une multiplication des acteurs et des interactions croissantes entre les niveaux de gouvernement (Theys, 2003). Assis sur plusieurs lois (1964, 1976, 1984, 1992), sur la Directive Cadre sur l'Eau (2000) et sa transposition française (2004), les docu-

9. AD29, 29S44, Avis de l'Ingénieur en Chef, 27 février 1946. 
ments de planification promeuvent progressivement la libre circulation comme critère d'amélioration de la qualité écologique et incitent les acteurs locaux à réaliser des aménagements pour le franchissement. Les politiques publiques s'orientent vers des pratiques d'effacement des ouvrages à partir de 2006. Sur l'Aulne, la restauration temporaire de la continuité écologique est expérimentée depuis 2010 en faveur des poissons migrateurs, au premier rang desquels le saumon, suscitant des controverses et des mouvements d'opposition plus ou moins organisés.

\section{Qualité biologique et continuité écologique comme nouvelles normes d'action et de gestion}

La loi sur l'eau de $1964^{10}$ marque l'émergence du modèle de la «nature milieu ». Le cours d'eau est un lieu de vie pour les espèces, l'eau est appréhendée peu à peu comme un hydrosystème (Aspe et Point, 1999). La politique de l'eau s'appuie sur des normes de rejets pour limiter la pollution de l'eau et des milieux. En Bretagne, l'Association pour la Protection et la Promotion du Saumon en Bretagne (APPSB) est créée en 1969 et fait de la présence du saumon atlantique un symbole de la qualité de l'eau continentale. Connue pour son slogan «Quand le poisson meurt, l'homme est menacé », l'association devient un acteur incontournable de la protection de l'eau et de la restauration de sa qualité à l'échelle régionale. À l'échelle nationale, la gestion du stock de saumons s'organise autour du premier Plan Saumon lancé en 1976 suite à la loi relative à la protection de la nature ${ }^{11}$ qui reconnaît les espèces animales comme partie intégrante du patrimoine naturel. C'est le premier programme d'action spécifiquement dédié à l'espèce en France. Il prévoit des réalisations en matière de libre circulation piscicole et de déversement de juvéniles (Dartiguelongue, 2012). Une modification de la catégorisation de l'espèce s'opère avec la loi sur la pêche de 1984, qui qualifie le poisson de «bien commun ». Cette loi institue l'obligation d'équiper par des dispositifs de franchissement les barrages situés sur les cours d'eau classés en cours d'eau à migrateurs par le décret du 31 janvier 1922. Des débits réservés sont instaurés au droit des ouvrages.

10. Loi n 64-1245 du 16 décembre 1964

11. Loi n ${ }^{\circ}$ 76-629 du 10 juillet 1976.
À partir de 2004, l'action publique est orientée sur la reconquête de la qualité de l'eau pour répondre aux objectifs émis par la DCE. Alors que dans les années 1960 la préservation de la qualité de l'eau avait comme socle des normes d'émission à partir de l'étude de la composition chimique de l'eau, la référence écologique entraine le développement de normes d'immission (Barraqué, 2004). Les conditions de référence d'un système écologique sont celles d'une absence ou quasi absence de perturbations anthropiques (Bouleau et Pont, 2014). La qualité écologique est évaluée à partir de différents paramètres : la biologie, les éléments hydromorphologiques construits sur le régime hydrologique, la continuité de la rivière et la morphologie, et enfin les éléments physicochimiques; ces derniers étant des paramètres anciens et fondamentaux de la caractérisation de la qualité de l'eau. L'abondance et la richesse spécifique des écosystèmes sont les principaux paramètres de l'évaluation de la qualité biologique ce qui conduit à préserver et restaurer certains compartiments, certaines fonctions, certaines espèces plutôt que d'autres. La fréquentation des axes fluviaux par les poissons migrateurs est un critère de l'évaluation qui a mené à une prise en compte croissante de l'ensemble des espèces migratrices. Les bioindicateurs deviennent des outils de l'évaluation qui déterminent la proximité ou non avec la norme du bon état écologique (Reyjol et al, 2012).

Selon ces objectifs de nature écologique, la loi sur l'eau et les milieux aquatiques de $2006^{12}$ réaffirme la nécessité de restaurer la continuité écologique, elle introduit ainsi « une relation fonctionnelle entre d'une part aménagement et morphologie des cours d'eau et d'autre part leur état écologique » (Bellec et Lefebvre, 2012, p. 10). La continuité écologique devient un élément de la qualité ${ }^{13}$ en 2010 . La formulation d'objectifs ambitieux et l'approche écosystémique des cours d'eau augmentent les linéaires concernés par les classements pour la mise en conformité avec les objectifs de continuité (Bellec et Lefebvre, 2012). L'effacement des ouvrages transversaux est alors une mesure privilégiée par l'État français et reflète la domination d'une nouvelle doctrine d'aménagement des cours d'eau (Barraud,

12. Loi n 2006-1772 du 30 décembre 2006

13. Arrêté du 25 janvier 2010 relatif aux méthodes et critères d'évaluation de l'état écologique, de l'état chimique et du potentiel écologique des eaux de surface. 
2011). Les listes 1 et 2 issues de l'article L.214-17 du Code de l'Environnement remplacent le classement en cours d'eau réservés et classés (ONEMA, 2011). Sur les portions de cours d'eau identifiées au titre de la liste 2 , les ouvrages transversaux doivent être mis en conformité avec la continuité écologique dans les cinq ans suivant la publication de l'arrêté préfectoral. L'aménagement écologique par le démantèlement des ouvrages permet d'intervenir simultanément sur la qualité de l'eau et sur le peuplement piscicole. Ainsi, la libre circulation est une action qui tend à être privilégiée dans les recommandations des organismes internationaux de protection des poissons migrateurs. Des textes français et européens tels que les lois issues du Grenelle de l'Environnement et le Plan de gestion de l'Anguille en 2010 visent à limiter la fragmentation des habitats par une reconnexion des réservoirs biologiques. En Bretagne, la continuité écologique répond principalement aux objectifs de restauration et de protection des poissons migrateurs. Le SDAGE 20102015 Loire-Bretagne identifie la réouverture des cours d'eau aux poissons migrateurs comme une disposition fondamentale, si bien que l'Agence de l'eau Loire-Bretagne finance en priorité les effacements d'ouvrages.

\section{La restauration de la population de saumons sur l'Aulne}

À l'échelle française, plusieurs éléments caractérisent la politique de protection et de restauration des poissons migrateurs à partir des années 1970 (Dartiguelongue, 2012). On peut les regrouper en cinq catégories d'action. (1) Le passage du tout saumon vers la prise en compte de l'ensemble des espèces dites grands migrateurs (amphihalins) est acté. Dès 1981, le Premier plan quinquennal poissons migrateurs (1981-1985) est mis en œuvre. (2) Les plans poissons migrateurs sont intégrés aux Contrats de Plan Etat-Région en 1982. La contractualisation devient le mode privilégié de gestion et permet l'accélération de la mise en œuvre (Larrue, 2014). (3) En 1994, la création des COGEPOMI (Comités de Gestion des Poissons Migrateurs) et des PLAGEPOMI (Plans de Gestion des Poissons Migrateurs) intégrés aux CPER consacre la régionalisation de la gestion. (4) Les démarches incitatives sont progressivement remplacées par des obli- gations de résultats. (5) La mise en œuvre d'une gestion patrimoniale de la ressource est au service du développement de populations de saumons sauvages autonomes. Les actions de soutien d'effectifs perdent de l'importance au profit de mesures d'aménagement des ouvrages et de réglementation de la pêche.

Sur le bassin versant de l'Aulne, les mesures prises pour restaurer et protéger la population de saumons représentent un effort financier très supérieur à celui consenti pour les autres espèces migratrices. Par ailleurs, c'est sur ce cours d'eau breton que se concentre l'effort financier régional. La libre circulation constitue en moyenne $24 \%$ des dépenses dans les différents plans et arrive en deuxième position après le soutien d'effectifs (Dartiguelongue, 2012). La succession des plans migrateurs depuis 1975 a conduit à l'équipement de 21 dispositifs de franchissement par le Syndicat mixte d'aménagement touristique de l'Aulne et de l'Hyères (SMATAH) en charge des travaux pour le compte de la Direction éépartementale de l'Équipement. Des rénovations de passes à poissons sont réalisées sur certains ouvrages afin d'en améliorer la franchissabilité. Sur les barrages non équipés, des passes à poissons « nouvelle génération » sont construites. Des dispositifs datant des années 1950-1960 sont conservés sur 7 barrages. Les campagnes des années 1990 visent à compléter l'équipement ou le rénover. Une des caractéristiques principales de la politique d'équipement de cette période est la relative absence de réflexion aval-amont dans la construction ou la modification des passes à poissons et la coexistence de différents systèmes de franchissement à l'efficacité variable (figure 3). Sur le site de Coatigrac'h ( $3^{\mathrm{e}}$ ouvrage aval), la passe à poissons constitue un point noir pour le franchissement des différentes espèces au début de leur parcours de migration, elle est cependant reconstruite à l'identique. De plus, la majorité des dispositifs ont été pensés pour le franchissement spécifique du saumon, seule une passe sur l'ensemble du parcours est adaptée à toutes les espèces de migrateurs.

Les campagnes réalisées dans le cadre des plans poissons migrateurs ont abouti à la réalisation d'un dispositif de franchissement sur chaque barrage de l'Aulne canalisée. Mais à l'image de la période précédente (années 1860-années 1930), les campagnes de travaux menées entre 1950 et 2000 n'ont pas 


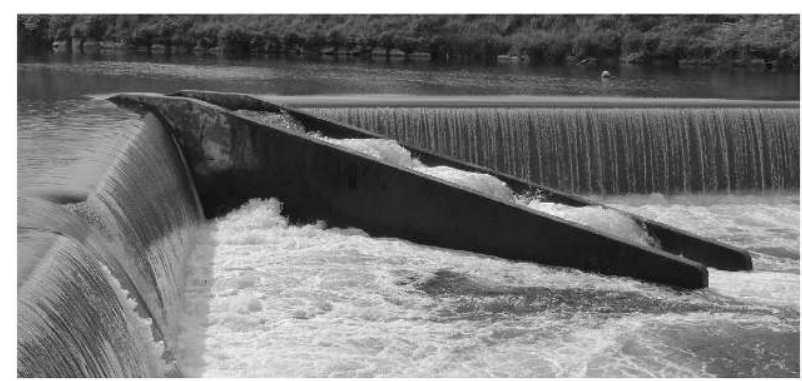

Echancrure et passe à bassins à parois déversantes - Prat Pourric

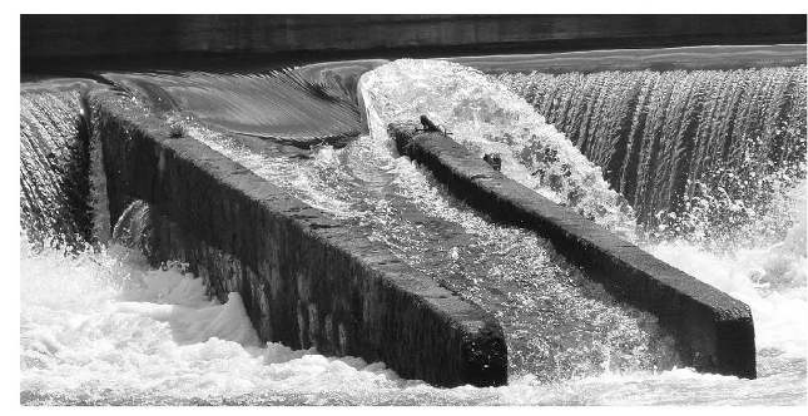

Rampe à canoës - Kersalic

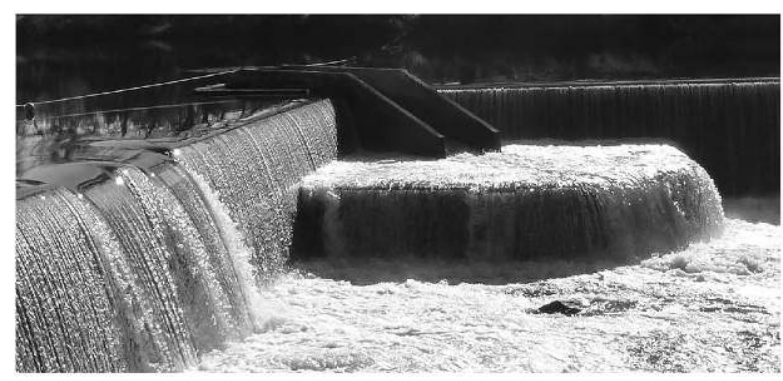

Echancrures et passe à ralentisseurs mixte poissons-canoës avec pré-barrage - Toul ar Rodo

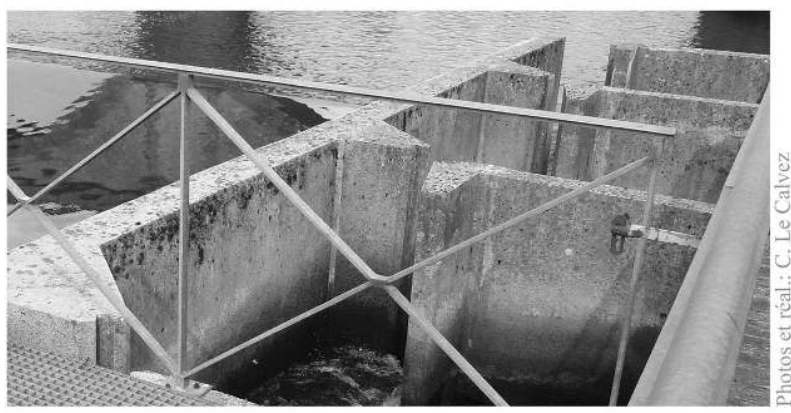

Passe à bassins à fentes verticales - Châteaulin

Figure 3 : Les dispositifs de franchissement sur l'Aulne canalisée (crédits : C. Le Calvez, 2014) Fishways on the canalised Aulne (credits : C. Le Calvez, 2014)

rempli leur objectif de restauration d'une population de saumons autonome sur l'Aulne.

\section{Ouvrir les pertuis des barrages : un compromis souhaitable?}

À la fin des années 1990, le constat d'une dégradation généralisée de l'état du canal fait réagir les acteurs locaux. La voie d'eau n'est presque plus empruntée sur l'ensemble de la section finistérienne faute d'entretien suffisant des aménagements, la pollution de l'Aulne est perçue comme problématique par les acteurs locaux. Pour les pêcheurs de saumon, la mauvaise qualité de l'eau est citée comme la principale cause limitant la qualité de la pêche devant l'impact de la canalisation (Salanié et al, 2004). La réflexion du SAGE Aulne est lancée en 2001 autour des enjeux de la production d'eau potable, du fort potentiel de production de saumons et des paysages « de grande qualité ». Deux projets de développement local opposés voient le jour, l'un organisé autour de l'Aulne comme voie de navigation fluviale et patrimoniale, l'autre en proposant de supprimer son caractère canalisé. L'image d'un canal navigué reste cependant ancrée dans les esprits. Le paysage fluvial et les usages de loisirs associés portent les nouveaux enjeux du développement local (Guibert et Paul, 2013). Les aménagements historiques de l'infrastructure fluviale (écluses, maisons éclusières, chemin de halage...) sont au centre d'un projet de patrimonialisation et d'une mise en valeur touristique par le SMATAH dans une logique d'aménagement du territoire. Cette politique qui émerge à la fin des années 1990 coïncide avec un projet de débarrage définitif qui remet en cause l'existence du canal. Portée par la Fédération de Pêche et soutenue notamment par Eau et Rivières de Bretagne, cette suppression des barrages repose sur un argumentaire écologique : il s'agit d'apporter une réponse efficace à la problématique du saumon dans l'Aulne, améliorer la qualité paysagère de la vallée et retrouver une eau de qualité (Eau et Rivières de Bretagne, 1997).

Dans le cadre du contrat "Retour aux sources" intégré au CPER 1995-1999, une étude de radiopistage de saumons visant à observer le comportement des saumons lors de la remontée est commanditée conjointement par la Fédération de Pêche du Finistère et le Conseil Supérieur de la Pêche. Elle renforce l'orientation vers le réaménagement des ouvrages du canal en démontrant que malgré 
l'équipement de la totalité des barrages en dispositifs de franchissement, seuls 3 à $4 \%$ des saumons suivis lors de l'étude parviennent sur les zones de frayères à l'amont de la partie canalisée (Croze et al., 2001). L'arasement des ouvrages ou des débarrages est préconisé par des experts pour améliorer le taux d'accès des saumons sur les zones de reproduction (Porcher et Ledouble, 2005). Dans une perspective inverse, l'Aulne canalisée est classée en 2007 en Masse d'Eau Fortement Modifiée par le Comité de bassin de l'Agence de l'Eau Loire-Bretagne. Cette décision entérine la sauvegarde du caractère canalisé. Les aménagements doivent être conservés au détriment des objectifs de qualité écologique. Seul le bon potentiel écologique de la masse d'eau est demandé. Puis en 2010, malgré la nécessité recon- nue 3 ans auparavant de sauvegarder les aménagements du canal, le programme du site Natura 2000 « la vallée de l'Aulne » réaffirme l'objectif de garantir la circulation des poissons migrateurs. La même année les acteurs locaux réunis au sein du Comité de pilotage Natura 2000 planifient une expérimentation de restauration temporaire de la continuité écologique. Conçue comme une synthèse entre les enjeux de la valorisation touristique et les objectifs écologiques, elle consiste en une onde de migration temporaire et progressive aval-amont par l'ouverture des vannes des pertuis situés sur les barrages (figure 4). Elle est réalisée deux fois par an au printemps et à l'automne pour coïncider avec le cycle de migration des saumons et favoriser leur remontée sur l'Aulne rivière qui concentre les surfaces de reproduction.

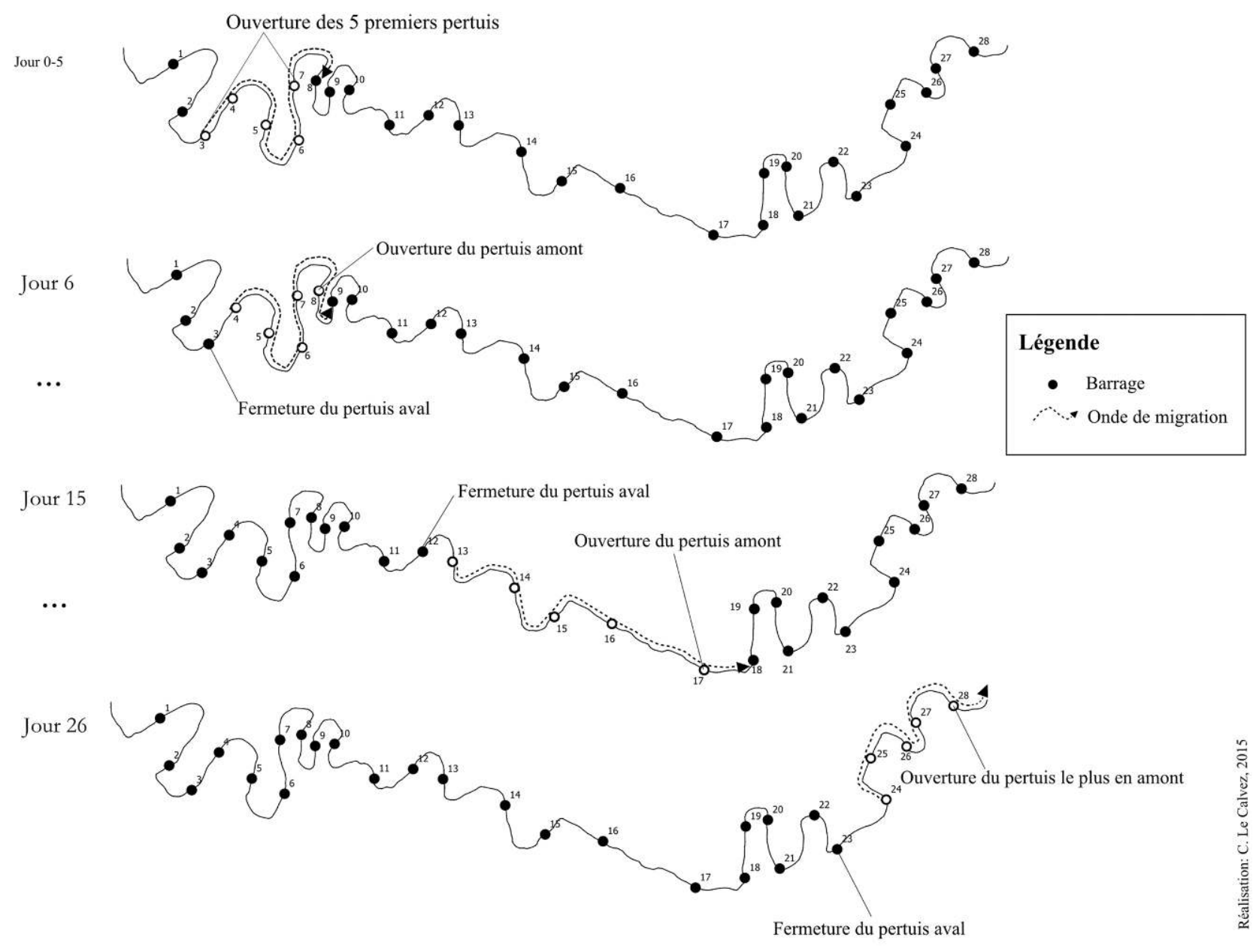

D'après Porcher et Ledouble (2005) et EPAGA

Figure 4 : Protocole expérimental d'ouverture des vannes de pertuis (2010) Experimental protocol for opening valves (2010) 
L'objectif de l'ONEMA, de la Fédération de Pêche, du Conseil départemental et de l'Établissement Public d'Aménagement et de Gestion du bassin versant de l'Aulne (EPAGA) est de statuer sur l'efficacité de la démarche. Son intérêt est de répondre temporairement aux objectifs Natura 2000 pour la libre circulation des espèces de migrateurs et de restaurer la continuité écologique, un des objectifs du SAGE Aulne. Cette expérimentation reste principalement ciblée sur l'enjeu piscicole.

L'approche du cours d'eau est renouvelée. Le projet de sauver le saumon de l'Aulne se poursuit à la fois parce que l'espèce est emblématique du territoire et parce que sa présence est le reflet d'un milieu en bonne santé. La disparition du transport fluvial a engendré la suppression de la voie d'eau comme infrastructure économique et une modification de sa gestion. Depuis les années 1990, la section canalisée de l'Aulne est valorisée dans le cadre du tourisme vert. À cette orientation s'ajoute la volonté d'en faire un cours d'eau qui réponde aux nouvelles normes de qualité écologique. L'intervention sur les milieux reste contrainte par l'existence de l'infrastructure, défendue par un certain nombre d'intérêts locaux.

\section{L'Aulne CANALISÉE À la CROISÉE DES ATTENTES : ENTRE VALORISATION DU PATRIMOINE HISTORIQUE ET RESTAURATION DU PATRIMOINE NATUREL}

L'Aulne constitue encore aujourd'hui un site majeur de mise en valeur de la ressource en saumon à l'échelle française : les captures réalisées représentent jusqu'à $1 / 3$ des captures nationales certaines années (Dartiguelongue, 2012). Or, l'injonction récente de restauration de la continuité écologique de ce cours d'eau très aménagé, portée par une action publique de nature réglementaire et normative, engendre des bouleversements déterminants dans la manière de penser localement le cours d'eau dans la vallée et dans un territoire. L'ancrage de la continuité écologique dans le projet territorial est une condition nécessaire à sa mise en œuvre, et force est de constater que ce processus d'ancrage n'est ni maîtrisé ni abouti. Très profondément, l'expérimentation d'ouverture des pertuis a déclenché la manifestation de blocages sociaux, avec la mobilisa- tion de groupes rétifs à la négociation et au compromis; s'y cristallisent des clivages politiques et idéologiques plus généraux sur la légitimité de l'application locale de politiques publiques nationales et européennes. In fine, derrière cette confrontation de visions territoriales, se pose la question de la préservation des «patrimoines » et des «paysages » construits, représentés par le canal et ses aménagements d'une part, par les espèces migratrices et leur écosystème d'autre part.

\section{Quelle demande sociale pour quel projet de territoire?}

L'expérimentation qui se déroule sur la partie canalisée est l'objet d'une controverse entre les acteurs de la mise en œuvre (figure 5). Elle a engendré un conflit impliquant des usagers du canal opposés au débarrage expérimental, réunis au sein de l'Association de Sauvegarde de l'Aulne Canalisée (ASAC) depuis 2013. S'exprimant dans les médias et lors des réunions du comité d'usagers de l'expérimentation organisées par l'EPAGA, ces usagers aux intérêts diversifiés s'opposent à la mise en œuvre de cette expérimentation pilotée par des acteurs institutionnels et associatifs. Ils nient l'efficacité de l'opération pour la restauration du saumon et l'accusent de détruire l'écosystème en place depuis la création du canal pour favoriser une espèce qui n'est quasiment plus pêchée. Ils expriment la crainte que cette expérimentation soit un coup d'essai avant une suppression définitive du canal, si bien que la méfiance vis-à-vis des intentions implicites des politiques publiques constitue un levier et un ciment puissant de l'opposition. Le projet porté par l'expérimentation repose sur une expertise écologique qui fait de la biodiversité un outil de promotion de la vallée de l'Aulne. Les dimensions socioéconomiques demeurent les grandes absentes du projet et la justification écologique ne suffit pas à ce que l'expérimentation soit appropriée. Le rejet manifesté par les opposants de l'ASAC (prises de position médiatiques, enchaînements d'opposants aux pertuis pour empêcher leur ouverture, manifestations lors des ondes de migration) pose la question du projet de société et de territoire que construisent l'expérimentation et le retour du saumon. Or l'inclusion dans un projet de territoire apparaît comme un préalable à son appropriation par la société locale 


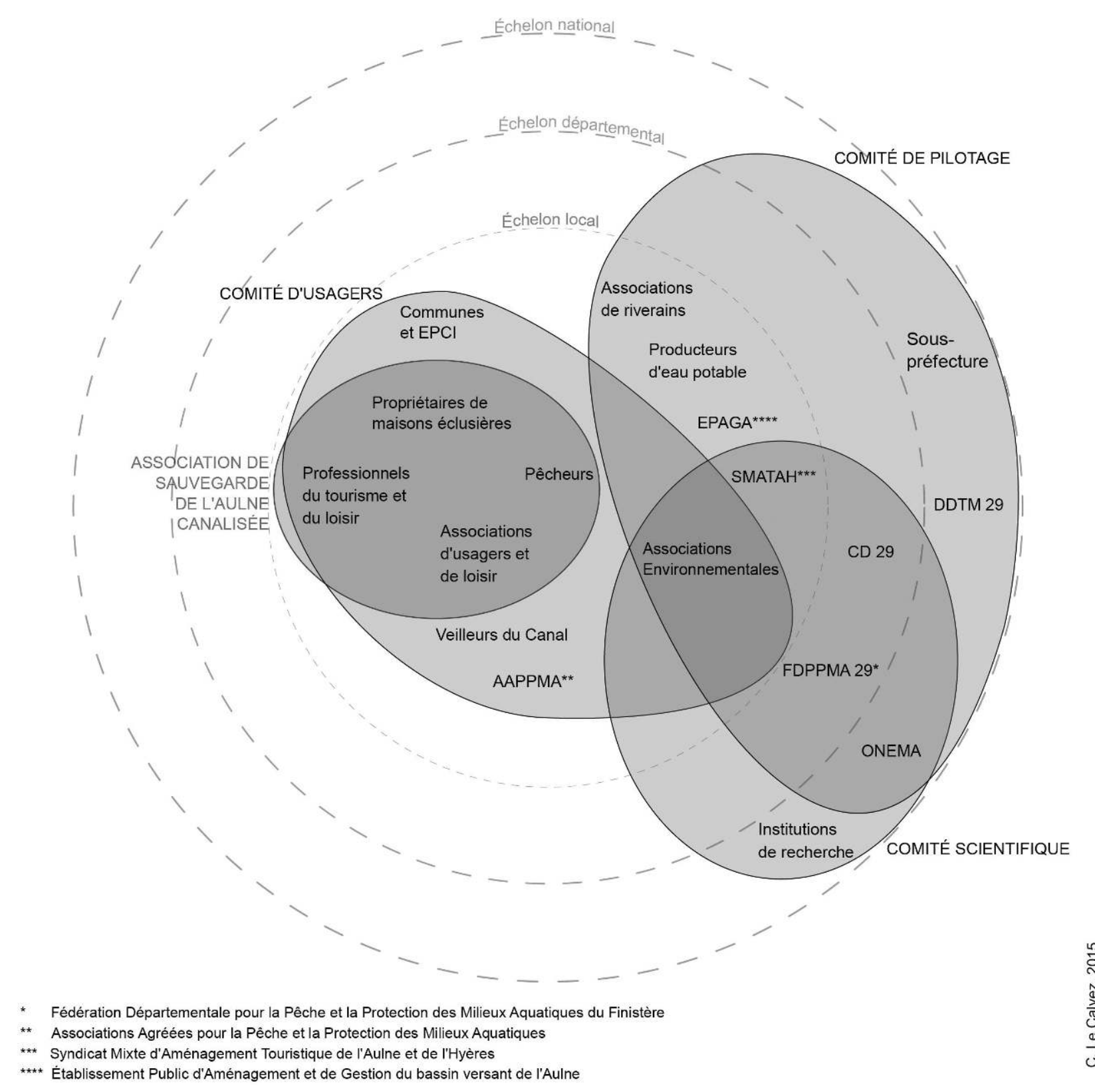

Figure 5 : Les acteurs de la mise en œuvre de l'expérimentation Actors in the implementation of the experiment

(Germaine, 2011; Germaine et Barraud, 2013; Germaine et Barraud, 2014). Pour le groupe d'opposants, la protection doit prioritairement porter sur le patrimoine historique (Kalaora, 1998) pour conserver la voie d'eau et le paysage associé, patrimoine où le saumon ne semble pas un élément central.

Tel qu'il est pensé et mis en ouvre, le protocole d'ouverture des pertuis reste une intervention technique sur le cours d'eau portée par un modèle d'aménagement environnementaliste normalisé. L’ingénierie écologique doit cependant prendre en compte la complexité des effets socio-spatiaux qui en découlent, car ces interventions requalifient les espaces de vallées (paysages, usages) et engendrent des mutations sensibles dans les relations symboliques et identitaires au territoire (Charles et Kalaora, 2003). Une réflexion sur le devenir de la vallée dans son ensemble doit être menée en invi- 
tant à poser la question de l'articulation entre les exigences de qualité écologique et les attentes des populations. La difficulté est bien celle de la nécessaire conciliation des exigences réglementaires en matière d'environnement avec les enjeux du territoire d'application. Les objectifs de qualité de l'eau et des milieux aquatiques tels qu'ils sont formulés dans les documents d'objectifs et de planification ne sont pas lisibles dans une perspective de développement socio-territorial. Un exemple peut être pris avec la politique de développement touristique de la vallée menée par le gestionnaire. Aucune passerelle entre l'écologie et le tourisme n'est construite, l'expérimentation est au contraire perçue comme un frein pour l'activité touristique pour le gestionnaire et les opposants exerçant une profession dans ce domaine. Enfin, l'effort financier est centralisé, porté entièrement par l'Europe et l'État à travers le ministère de l'Écologie pour les barrages situés au sein du périmètre Natura 2000. Cette absence d'implication financière des échelons locaux (collectivités et leurs regroupements) engendre une forme de déconnexion avec le territoire. Seule l'ouverture de 3 pertuis en dehors du site de la «vallée de l'Aulne » est financée par le gestionnaire, la collectivité et l'Agence de l'eau. L'expérimentation est développée comme une émanation de la politique européenne et nationale, que l'on pourrait qualifier de « hors-sol », d'autant plus exogène qu'elle n'a pas été l'occasion d'une concertation sur sa justification locale avec l'ensemble des acteurs. Actuellement, la reconquête du saumon sur l'Aulne est dissociée des questions économiques et touristiques qui étaient un principe et moteur de l'action au début $\mathrm{du} \mathrm{Xx}^{\mathrm{e}}$ siècle. Ainsi, en un siècle de politique en faveur de la remontée des saumons, un basculement s'est opéré dans la justification de l'aménagement des ouvrages. Alors que le caractère expérimental pourrait être un moment privilégié de réflexion et de détermination d'un projet partagé, le processus d'ouverture des pertuis reste une intervention confidentielle, impliquant matériellement quelques animateurs-techniciens et usagers intéressés.

\section{D'une conception de l'Aulne à une autre}

Depuis le milieu du xIX $^{\mathrm{e}}$ siècle, la difficulté à laquelle font face les acteurs locaux est bien celle de parvenir à faire de l'Aulne canalisée une rivière à saumons tout en préservant les caractéristiques de la rivière aménagée. Les politiques de protection et de restauration de la ressource en saumons ont tenté de répondre à cette difficulté en équipant tous les barrages puis en les effaçant. Sur chaque ouvrage une succession voire une superposition de dispositifs et de mesures de franchissement a été appliquée (figure 6). L'Aulne a ainsi d'abord été perçue lors d'une première phase comme un axe fluvial qui renvoie au modèle de la rivière aménagée pour les besoins humains. Le cours d'eau est un espace productif avec lequel l'enjeu du saumon doit composer, l'équipement est une solution consensuelle. La $2^{\text {e }}$ phase est celle de l'Aulne « milieu » qui correspond à la rivière aménagée comme lieu de vie. On se situe dans un entre-deux où les aménagements n'ont plus de vocation économique sans pour autant être remis en cause. Dans un dernier temps, la gestion écologique du cours d'eau a conduit à exiger l'adaptation de l'infrastructure économique pour correspondre aux normes environnementales en vigueur sans statuer définitivement sur son devenir. La genèse de ce dernier type d'actions témoigne plus généralement de ce que Barraud (2011) a mis en exergue au sujet de la dynamique des modèles de nature et de paysage. Après une période d'équipement et de ré-empoissonnement, solutions techniques et artificielles, des méthodes considérées comme plus «naturalisantes » sont mises en place et coïncident avec la montée en puissance du modèle esthétique et des valeurs associées à la nature sauvage (Barraud, 2011). Le cours d'eau doit divaguer, il est une infrastructure naturelle en rupture avec la rivière de pleins bords symbole du modèle aménagiste antérieur (Germaine et Barraud, 2014).

À bien des égards, l'effacement des ouvrages, même temporaire, se distingue de l'équipement en passes à poissons par son impact socio-spatial. Il entraîne une transformation fonctionnelle du cours d'eau avec une diversification des écoulements. La baisse du niveau de l'eau induit des changements paysagers en fond de vallée. Les usages de l'eau doivent s'adapter à la nouvelle configuration du cours d'eau. Ce nouveau mode d'aménagement entre en tension avec le paysage hérité auquel la population locale est attachée, tensions que les discours et les prises de position des acteurs de l'opération retranscrivent. D'après nos observations et nos enquêtes, la pensée des écologues, biologistes et 


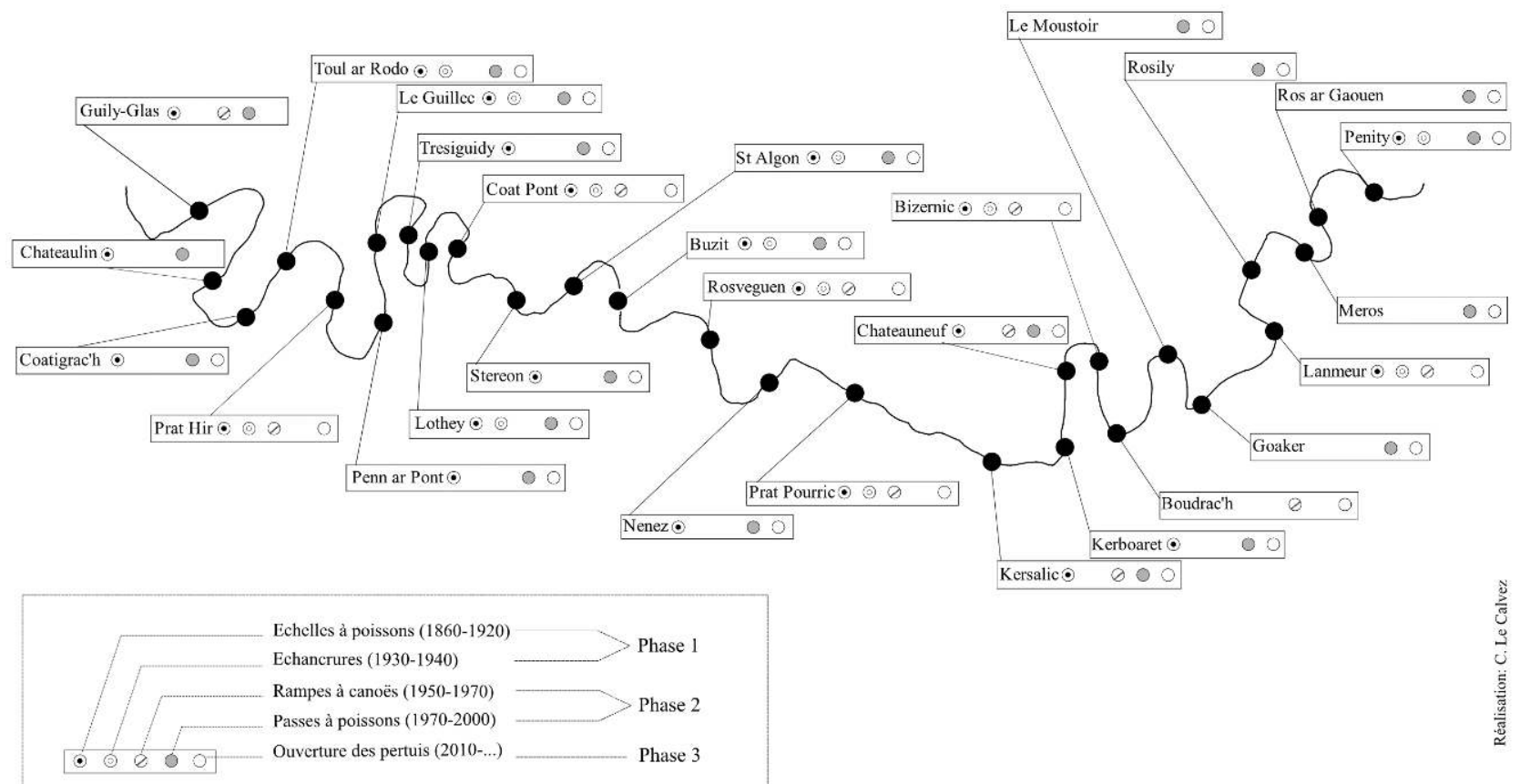

Sources: AD 29, ONEMA, Fédération de Pêche du Finistère

Figure 6 : La trajectoire d'aménagement des barrages sur l'Aulne canalisée Evolution of dam management the canalised Aulne

hydrologues tend à plaider pour la qualité des conditions d'épanouissement des espèces migratrices en l'absence d'ouvrages, tout en restant incertains sur l'effet potentiellement contre-productif de la restauration des débits sans barrages. Les riverains, l'organisme de gestion du canal tendent à considérer le canal comme un écosystème de qualité, avec ses rythmes, ses caractéristiques paysagères, son histoire, ses espèces de poissons blancs. Pour ces acteurs locaux, dont font partie certains élus, le saumon doit transiger avec l'existant, alors que la politique publique portée par le Conseil départemental, l'EPAGA et la Fédération de Pêche vise à l'inverse à adapter la voie d'eau au saumon sans pour autant approuver les propositions de débarrage définitif qui avaient été formulées à deux reprises au siècle précédent. En ce sens, la prise de position contre le retour à un état naturel relève bien d'une vision du territoire qui ne se pense pas en dehors de la pérennisation du caractère canalisé de l'Aulne.

\section{Conclusion : l'Aulne DANS UN ENTRE-DEUX}

La restauration de la population de saumons est un enjeu qui a traversé le $\mathrm{xx}^{\mathrm{e}}$ siècle sur l'Aulne. La dynamique des acteurs initiateurs, l'évolution des justifications, l'évolution de la perception de l'enjeu façonnent la politique d'aménagement des ouvrages transversaux. En définitive, la dimension écologique attribuée aux cours d'eau donne aux poissons migrateurs un nouveau rôle, celui d'indicateur de la qualité de l'eau et des milieux aquatiques. La loi de 1984 sur les dispositions de franchissement des ouvrages par les grands migrateurs a constitué un premier tournant «écologique » de la gestion des cours d'eau (Germaine et Barraud, 2013). La perception de l'enjeu poisson migrateurs dans lequel le saumon figure à la première place, ne s'appréhende plus uniquement par l'entrée de la quantité de poissons dans une démarche de restauration des stocks, mais est investie d'une dimension qualitative qui devient dominante. Cette évolution du sens de leur présence conduit à une transformation des modes 
d'interventions. L'équipement en passes à poissons, qui a été une action privilégiée pendant plus d'un siècle pour recréer la libre circulation n'est plus la solution incontournable. Sur l'Aulne, l'efficacité de ces dispositifs n'a pas été à la hauteur des attentes et des efforts financiers consentis. La mise en œuvre de la continuité écologique par l'arasement ou l'effacement des ouvrages transversaux se présente comme un nouvel outil technique séduisant mais radical entre les mains des acteurs, à la croisée des enjeux de la biodiversité et de la qualité de l'eau. Pour aller au-delà des dispositifs de franchissement et par impossibilité de mettre en place la solution de la suppression définitive des barrages, l'ouverture des pertuis menée sur l'Aulne canalisée depuis 2010 peut être considérée comme une synthèse expérimentale des attentes réglementaires en matière de milieux aquatiques et de gestion des espèces migratrices. La recherche de la continuité de la rivière pour le saumon emprunte ainsi les outils développés pour restaurer la continuité écologique des cours d'eau, à savoir l'effacement des obstacles, mais sans véritable perspective d'amélioration globale de la biologie et de l'hydromorphologie dans le cas développé.

Par ailleurs, d'autres expériences sont menées dans l'Ouest de la France, comme le projet de double effacement d'ouvrages hydroélectriques sur la Sélune. La reconstitution par Germaine et Lespez (2014) de la trajectoire de valorisation de la Sélune autour des barrages de la Roche-qui-Boit et de Vezins témoigne de l'articulation récente entre l'effacement d'ouvrages et le repeuplement des vallées fluviales en poissons migrateurs. En effet, la construction des barrages sur la Sélune a permis une valorisation par l'exploitation énergétique et le développement touristique autour des lacs de retenue. L'existence de ces usages de l'eau est aujourd'hui interrogée par la valorisation écologique (Germaine et Lespez, 2014). À nouveau, le caractère technique du projet d'effacement, qui se limite à la reconquête du fleuve pour une espèce emblématique, est mis en exergue et critiqué. À l'inverse, l'élargissement des problématiques de continuité écologique aux attentes des usagers, aux dynamiques des territoires par le redéploiement d'usages peut représenter des opportunités de développement territorial. Le maintien de l'attractivité par le tourisme pêche, la valorisation d'une qualité environnementale aujourd'hui recherchée par les populations sont autant de propositions dont peuvent s'emparer les acteurs. Entre patrimonialisation des activités humaines et patrimonialisation de la nature, l'effacement des ouvrages questionne le devenir des usages et des paysages fluviaux. Il légitime un paysage fluvial naturel au sein de coulées vertes et appelle certains usages (tourisme vert, tourisme pêche...) au détriment d'usages traditionnels comme la navigation ou la pêche au coup qui sont associées à un paysage aux motifs humains dominants.

Les prises de positions favorables ou à charge font glisser ces projets initialement écologiques vers une réflexion sur le devenir socio-économique des territoires. Ils ne peuvent faire l'économie d'une appréhension par le politique. Ainsi, les controverses qui se développent conduisent à penser que la restauration de la continuité écologique doit sortir des cercles scientifiques et techniques et dépasser l'approche écologique dominante qui conduit à faire des cours d'eau des linéaires déterritorialisés. C'est au prix d'une coordination avec les enjeux locaux, de l'appui sur des bases territoriales renouvelées et adaptées aux territoires locaux, que l'ancrage de cette mesure, en rupture avec les interventions traditionnelles, pourrait aboutir.

\section{Bibliographie}

Aspe C., Point P. (coord.), 1999. L'eau en représentations. Gestion des milieux aquatiques et représentations sociales, Cemagref Editions, GIP Hydrosystèmes, 101 p.

Arago M.-A., Vauclin V., 2000. Bilan du programme « poissons migrateurs » du contrat de plan État-Région 19941998 en Bretagne, Bulletin Français de la pêche et de la pisciculture, $\mathrm{n}^{\circ} 357-358$, p. 357-372.

Barraqué B., 2004. Normes de qualité de l'eau : histoire et prospective, $15^{\mathrm{e}}$ journées scientifiques de l'Environnement, Usages de l'eau : synergies et conflits, mai, Créteil, France, HAL, 14 p.

Barraud R., 2011. Rivières du futur, wild rivers?, VertigO - la revue électronique en sciences de l'environnement [en ligne], hors-série $n^{\circ}$ 10, DOI : 10.4000/vertigo.11411.

Barraud R., Portal C., 2013. Les petites vallées des Pays de la Loire. Du paysage pittoresque aux nouvelles interprétations patrimoniales : permanence et tensions contemporaines, Norois, $n^{\circ} 228$, p. 11-26. DOI : 10.4000/norois.4717.

Bellec P., Lefebvre E., 2012. Plan d'actions pour la restauration de la continuité écologique des cours d'eau (Parce), 
Conseil Général de l'Environnement et du Développement Durable, 78 p.

Bouleau G., Pont D., 2014. Les conditions de référence de la directive cadre européenne sur l'eau face à la dynamique des hydrosystèmes et des usages, Natures Sciences Sociétés, Vol. 22, n 1, p. 3-14.

Breton B., 1993. La pêche en France, Paris, PUF, coll. «Que sais-je? », 127 p.

Brunot A., Cocquand R., 1982. Le corps des Ponts et Chaussées, Paris, CNRS, 915 p.

Cambry J., 1993. Voyage dans le Finistère, Spézet, Coop Breizh, $479 \mathrm{p}$.

Chaney E., 1995. Les saumons de la rivière Snake sur le bassin de la Columbia : menaces et espoirs, Actes du colloque Des saumons et des hommes, Brioude, décembre 1993, SOS Loire Vivante, Le Puy, p. 69-72.

Charles L., Kalaora B., 2003. L’ingénierie écologique entre écologie, technique et aménagement : des enjeux durables, in Lévêque C., Van der Leeuw S. (dir.), Quelles natures voulons-nous? Pour une approche socio-écologique du champ de l'environnement, Paris, Elsevier, p. 225-235.

Clewell A-F., Aronson J., 2010. La restauration écologique, Actes Sud, 338 p.

Croze O., Sénécal A., Woillez M., 2001. Suivi par radiopistage de la migration anadrome du saumon atlantique sur l'Aulne. Déplacements des individus au cours de la campagne de terrain 2000, Fédération du Finistère pour la Pêche et la Protection du Milieu Aquatique, 168 p.

Croze O., 2008. Impact de senils et barrages sur la migration anadrome du saumon atlantique (Salmo salar L.) : caractérisation et modélisation des processus de franchissement, Thèse de doctorat en Fonctionnement des Écosystèmes et Agrosystèmes, Université de Toulouse, 331 p.

Dartiguelongue J., 2012. Bilan des études et actions de restauration du Saumon Atlantique sur le bassin de l'Aulne (29), Rapport SCEA pour Bretagne Grands Migrateurs, 81 p.

Eau et Rivières de Bretagne, 1997. Et si on libérait l'Aulne canalisée?, Revue Eau et Rivières, $\mathrm{n}^{\circ}$ 102, décembre, p. 7-14.

Germaine M.-A., 2011. Dépasser l'enjeu piscicole, vers la définition d'une gestion concertée du cours d'eau et de ses berges. L'exemple de la vallée de la Touques (Calvados), Géocarrefour, vol.86, n³-4, p. 245-259.

Germaine M-A., Barraud R., 2013. Restauration écologique et processus de patrimonialisation des rivières dans l'Ouest de la France, VertigO - la revue électronique en sciences de l'environnement [En ligne], hors-série nº 16, DOI : 10.4000/ vertigo. 13583.

Germaine M.-A., Barraud R., 2014. Les rivières de l'ouest de la France sont-elles seulement des infrastructures naturelles? Les modèles de gestion à l'épreuve de la directivecadre sur l'eau, Natures Sciences Sociétés, vol.21, $\mathrm{n}^{\circ} 4$, octobre-décembre, p. 373-384

Germaine M.-A., Lespez L., 2014. Le démantèlement des barrages de la Sélune (Manche). Des réseaux d'acteurs au projet de territoire?, Développement durable et territoires
[En ligne], vol.5, $\mathrm{n}^{\circ}$ 3, mis en ligne le 5 décembre 2014 , consulté le 19 mars 2015, 21 p.

Guibert C., Paul A., 2013. Usages et intérêts différenciés des espaces maritimes et fluviaux patrimonialisés. Les cas de Saint-Gilles-Croix-de-Vie en Vendée et du canal de Nantes à Brest, Norois, n²28, p. 89-101.

Kalaora B., 1998. Au-delà de la nature l'environnement. L'observation sociale de l'environnement, Paris, L'Harmattan, coll. «Environnement », 199 p.

Larrue C., 2014. Analyser les politiques publiques d'environnement, L'Harmattan, coll. Logiques politiques, 207 p.

Laurent J.-L., 1995. Le troisième plan migrateur pour les saumons de l'axe Loire-Allier, Actes du colloque Des saumons et des hommes, Brioude, décembre 1993, SOS Loire Vivante, Le Puy, p. 96-97.

Minot E., 2000. Département. Conseil Général. Décentralisation. Tome 1, Histoire et perspectives d'avenir, Editions de La Lettre du Cadre Territorial, Voiron, 222 p.

Morandi B., 2014. La restauration des cours d'eau en France et à l'étranger : de la définition du concept à l'évaluation de l'action. Eléments de recherche applicables, Thèse de doctorat en Géographie, ENS de Lyon - Université de Lyon, vol.1, 407 p.

ONEMA, 2010. Pourquoi rétablir la continuité écologique des cours d'eau ?, Paris, Éditions de l'ONEMA, coll. «Sensibilisation aux politiques publiques », $26 \mathrm{p}$.

ONEMA, 2011. La révision des classements de protection des cours d'eau. Un outil en faveur du bon état écologique et de la biodiversité, Editions de l'ONEMA, Collection Sensibilisation aux politiques publiques, $28 \mathrm{p}$.

Porcher J.-P., Ledouble O., 2005. Propositions de programmes de gestion des biefs pour améliorer la circulation des poissons migrateurs sur l'Aulne, Rapport du Conseil Supérieur de la Pêche, 30 p.

Rainelli P., Thibault M., 1980. La fabuleuse richesse en saumons des rivières bretonnes d'autrefois, mythe ou réalité, Annales de Bretagne et des pays de l'Ouest, vol. 87, $\mathrm{n}^{\circ} 4$, p. 697-713.

Reyjol Y., Spyratos V., Basilico L., 2012. Bioindication : des outils pour évaluer l'état écologique des milieux aquatiques. Perspectives en vue du $2^{\mathrm{e}}$ cycle DCE - Eaux de surface continentales, synthèse des journées "DCE et bioindication » du séminaire "Méthode d'évaluation de l'état des eaux - situation et perspectives dans le contexte de la Directive Cadre sur l'Eau », avril 2011, Paris, 31 p.

Salanié J., Surry Y., Le Goffe P., 2004. La pêche au saumon dans le massif armoricain : état des lieux et mesure de l'effort de pêche, Bulletin Français de la pêche et de la pisciculture, $\mathrm{n}^{\circ} 375$, p. 35-70.

Theys J., 2003. La Gouvernance, entre innovation et impuissance, Développement durable et territoires [En ligne], dossier 2 : Gouvernance locale et Développement Durable, DOI : 10.4000/developpementdurable. 1523.

Thibault M., Vinot C., 1989. Les moulins à eau sur les cours d'eau à saumon atlantique de Bretagne. Évolution et diversification des implantations; modification de l'écosystème, Revue de Géographie de Lyon, vol. 64, n 4, p. 204-212. 
Thibault M., 1995. La rivière et l'homme : qualité des eaux courantes et activités humaines : l'exemple des rivières à saumon de Bretagne depuis l'ancien Régime, in Actes de conférences 1994, Université d'été des enclos et des Monts
d'Arrée, Des ressources et des hommes, Éditions Pays touristique des Enclos et des Monts d'Arrée, p. 22-57.

Thomas S., 2009. La vie du canal de Nantes à Brest dans le Finistère (1826-1914), Spézet, Éditions Keltia Graphic, 240 p. 